Theory of justice: beyond the national limits

\title{
La teoría de la justicia: más allá de los límites nacionales
}

\author{
Irene Ortiz Gala \\ Universidad Autónoma de Madrid \\ irene.ortizg@uam.es \\ DOI: https://doi.org/10.15366/bp2019.22.003 \\ Bajo Palabra. II Época. No 22. Pgs: 81-94
}

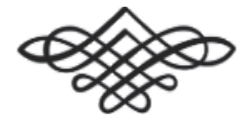


Recibido: 15/10/2016

Aprobado: 02/10/2019

\section{Resumen}

La teoría de la justicia generalmente trata problemas que se dan dentro de los límites nacionales, es decir, justicia e injusticia tienen en cuenta lo que pasa dentro de estos límites y a aquellos sujetos reconocidos como ciudadanos de ese país. Sin embargo, en los últimos ańos el debate de la teoría de la justicia se ha centrado no solo en qué es una injusticia sino también en quién debe ser tenido en cuenta como sujeto de justicia. Podemos entender como injusticia la exclusión por parte de las instituciones hacia aquellos sujetos a los que no consideran ciudadanos, haciéndoles imposible reclamar justicia. La pregunta que hacemos es: ¿cómo podemos establecer quién está legitimado como sujeto de justicia?

Palabras clave: Justicia, Globalización, Sujeto, Opinión pública.

\section{Abstract}

Theory of justice generally addresses issues within national borders, which means that justice and injustice only take into account what happens inside the borders of a country and those are recognized as citizen of that country. However, in recent years the debate around theory of justice has focused not only on what an injustice is, but also on who should be taken into account as subject of justice. Injustice could be also understood as the exclusion performed by institutions towards subjects who are not taken as citizens, making it impossible for them to claim justice. So the question is: how can we establish who is a legitimate subject of justice?

Keywords: Justice, Globalization, Subject, Public opinion. 
L a teoría de la justicia ha estado principalmente guiada por dos paradigmas en el siglo XX: redistribución y reconocimiento. Las reclamaciones redistributivas igualitarias han sido las principales demandas en torno a la justicia del último siglo. El acento se ha puesto en la dimensión económica, es decir, si en la esfera económica había una desigualdad que impedía a algunos ciudadanos participar en la esfera político-social, ésta debía ser solventada con ayuda del Estado. Este modelo lo hemos visto en muchas sociedades capitalistas europeas, cuyo modelo económico neoliberal se conciliaba con ayudas correctivas por parte del Estado. De este modo, el welfare state ha ido solucionando o corrigiendo las desigualdades que se producían como resultado del libre mercado. El Estado entendía que la desigualdad venía como resultado de la esfera económica, y era ahí donde concentraba todos sus esfuerzos orientados hacía políticas redistributivas que favorecieran la igualdad. Por otro lado, como desvelaba al comienzo del artículo, desde hace algún tiempo encontramos otro tipo de reclamaciones, en principio, también orientadas al Estado y enmarcadas en las reivindicaciones de justicia social: el reconocimiento. La política del reconocimiento ha subrayado que no todas las desigualdades provienen de la esfera económica y que, de hecho, hay reparaciones de justicia que tienen que hacerse desde la esfera social. "Aquí, el objetivo, en su forma más verosímil, es un mundo que acepte la diferencia, en el que la integración en la mayoría o la asimilación de las normas culturales dominantes no sea ya el precio de un respeto igual" ${ }^{1}$. Por lo tanto, modificar la subordinación de estatus, en el plano social, y de clase, en el plano económico, fueron dos ejes más o menos rígidos de la teoría de la justicia westfaliana (aunque pudiéramos distinguir entre enfoques monistas como el de Honneth y bidimensionales como Fraser).

Por otro lado, la teoría de la justicia que venimos presentando estaba enmarcada dentro de los límites del Estado nación. Esto suponía establecer la validez del análisis dentro de las fronteras de un determinado país. Así, los trabajos que encontramos al respecto a lo largo del siglo XX parecen mantenerse dentro del contorno dibujado por estos límites. El debate en torno al liberalismo político de Rawls y el republicanismo kantiano de Habermas, a pesar de las evidentes diferencias entre

\footnotetext{
Fraser, N., "La justicia social en la era de la política de la identidad: Redistribución, reconocimiento y participación”. ¿Redistribución o reconocimiento? Madrid, Morata, 2006, p. 17.
} 
ambas propuestas, mantenían ambos un punto en común: el espacio donde se desarrollaban sus teorías, a saber, el Estado-nación. Desde luego, en ambas teorías el Estado cumplía el papel de máxima institución de poder y tenía la capacidad de resolver los reclamos de justicia que se hicieran desde la opinión pública. Especialmente en la contribución de Habermas en torno a la capacidad de influencia de la opinión pública en las estructuras de gobernación, queda latente empero que la condición para participar es ser miembro reconocido, es decir, ciudadano, del Estado al que se pretende reclamar. Sin embargo, es interesante cómo el proyecto democrático de Habermas resalta lo que él mismo llama "la libertad de los antiguos", enfatizando la importancia del derecho de participación de los ciudadanos en la vida política.

La apuesta teórica y política de que a través del requisito de membresía se podría participar y tener un cierto control sobre las instituciones que nos gobiernan, de tal forma que "a través de tales procesos [participativos] el pueblo democrático se demuestra no solo el sujeto sino también el autor de sus leyes" ${ }^{2}$. En este sentido, la propuesta de Habermas es de relevante interés para el análisis de la teoría de la justicia en un mundo globalizado, pues la pérdida de participación en instituciones supranacionales anticipa el malestar de la ciudadanía en términos democráticos. Este punto se ha revelado especialmente sensible en los últimos ańos, constituyendo síntoma de ello los múltiples movimientos sociales que señalan la baja salud del proceso democrático. En este sentido, debemos indagar qué acontecimientos nos han llevado a perder la confianza en nuestros sistemas democráticos al mismo tiempo que las reclamaciones que se hacen piden más participación en la toma de decisiones. "Mientras que las formas de democracia siguen estando plenamente en su lugar $-\mathrm{y}$ hoy en algunos aspectos están realmente fortalecidas- la política y el gobierno están cayendo cada vez más en el control de las élites privilegiadas en la forma características de los tiempos pre-democráticos; y una de las principales consecuencias de este proceso es la creciente impotencia de las causas igualitarias" ${ }^{3}$. $\mathrm{Al}$ respecto encontramos diversos diagnósticos, pero, generalmente, con una raíz común: por un lado, la quiebra de la soberanía del Estado nación; por otro, la presencia de organizaciones supranacionales eximidas de cualquier tipo de control democrático. La erosión del principio de autogobierno nos lleva a repensar nuestras sociedades democráticas contemporáneas, donde, desde luego, los diferentes tipos de democracia entrarán en juego. Ambos problemas están profundamente interconectados en tanto en cuanto suponen que existen prácticas que configuran las

\footnotetext{
2 Benhabib, S., "Teoría discursiva y membresía política” Los derechos de los otros. Barcelona, Gedisa, 2005, p. 25.

3 Crouch, C., "Why Post-Democracy?" Coping with Post-Democracy, London, Fabian Society, 2000, p. 9.
} 
vidas de los ciudadanos, que desbordan los Estados territoriales y que, por lo tanto, deberían ser tomadas en cuenta a la hora de elaborar una teoría de la justicia. "A menudo, además, los problemas debatidos son consustancialmente transterritoriales y no pueden localizarse en un espacio westfaliano ni ser resueltos por un Estado westfaliano" ${ }^{4}$.

Existen, por lo tanto, problemas que no tienen una fácil ubicación en los límites territoriales de un Estado pero que, sin embargo, tienen consecuencias en la vida de los ciudadanos de uno o varios países. En este sentido, la teoría de la justicia, que mantenía como marco normativo al Estado-nación, necesita reformularse y tener en cuenta la incipiente globalización y las estructuras de gobernación supranacionales que afectan a su desarrollo. Frente a un Estado que podía resolver las injusticias que impedían la paridad participativa de sus ciudadanos en las instituciones, encontramos una constelación de organizaciones transnacionales que diluyen las responsabilidades de las reparaciones de las injusticias. "Parece que el sistema internacional basado sobre los Estados-nación se está transformando en un sistema político de autoridad sobrepuesta a múltiples niveles, con una baja diferenciación de funciones y una escasa legitimidad democrática" ${ }^{5}$. En este sentido, diremos, como sugiere Fraser, que la gramática de la discusión de la teoría de la justicia ha cambiado, haciéndose imposible mantener los marcos o las estructuras que se mantenían en las teorías normativas del Estado-nación. Dado que la disposición de la teoría de la justicia se ha visto afectada por la merma de soberanía de los Estados, parece legítimo preguntarnos: ¿Cuál es el marco adecuado que nos ha de permitir reflexionar sobre las exigencias de la justicia en un mundo en globalización?

Por un lado, debemos atender a que esta pregunta nos está remitiendo, en primer lugar, a la cuestión del enmarque de la propia teoría. Necesitamos volver a pensar cuáles son y cuáles deben ser los límites para examinar al sujeto de justicia bajo la luz de la globalización. Asimismo, debemos atender a las implicaciones que tiene para la teoría este descentramiento del sujeto de justicia, una vez que asumimos la superación de los límites que nos facilitaba el Estado-nación. "Las estructuras de gobernación, como no se limitan a los Estados, comprenden también organismos no estatales que generan reglas que han de aplicarse y que estructuran importantes área de interacción social" ". La cuestión del enmarque remite por tanto a las llamadas injusticias de segundo orden, resultado del des-enmarque de algunas personas que

\footnotetext{
${ }_{4}^{4}$ Fraser, N., "Transnacionalización de la esfera pública”, Escalas de justicia, Barcelona, Herder, 2008, p. 161.

5 Della Porta, D., "Globalizzazione e movimenti sociali: una introduzione", Globalizzacione e Movimenti Sociali, Roma, Manifestolibri, 2003, p. 9.

${ }^{6}$ Fraser, N., "Justicia anormal”, Escalas de justicia, Barcelona, Herder, 2008, p. 127.
} 
no son reconocidas como sujetos legítimos para reclamar la reparación de injusticias de primer orden. La gramática anterior de la justicia se centraba precisamente en este primer orden, es decir, atendía al qué de la justicia, ya fuera desde el plano económico a través de medidas redistributivas, o desde el plano social donde se insistía en la necesidad de un justo reconocimiento de todos los individuos. Ahora, diremos, nos encontramos ante un nuevo reto para la teoría, a saber, reformular el quién de la justicia y, en un tercer orden, el proceso por el cual debería establecerse el quién.

\section{Quién de la justicia}

Pensar QuiÉn es el sujeto en un determinado marco de justicia, nos lleva a hablar de ciudadanía y de quién otorga esta categoría. Lejos de hablar de la sociedad como universitas, es decir, de agrupación con un compromiso o empresa que persiguen un objetivo final que proporciona la naturaleza de la unión, las sociedades modernas se aglutinan como asociación civil. Rawls insistió en esta tesis en su artículo The Idea of an Overlapping Consensus, subrayando la necesidad de abandonar la idea de una comunidad política unida por una idea de bien. Efectivamente, la sociedad civil no elige libremente asociarse y constituir un régimen jurídico en base a una idea de vida buena o buen vivir. Sin embargo, podemos decir que esta asociación civil tampoco nace de un pacto entre individuos que aglutinan sus fuerzas para desarrollar sus intereses, metas o aspiraciones propias. El origen contractual propuesto por Rousseau o Hobbes no nos ayuda a entender en qué consiste una sociedad contemporánea, sino que, más bien, tenemos que partir del mito fundacional del pacto para establecer los límites de las comunidades y pensar desde ahí la categoría de ciudadanía. No obstante, cabría preguntarse qué es lo que hace que las sociedades se mantengan unidas, es decir, qué mecanismos se articulan en el seno de los Estados para que sus habitantes sean reconocidos como ciudadanos y, finalmente, como sujetos de justicia. Mouffe indica que lo que nos hace ciudadanos en un régimen democrático general es "estar asociados en función del reconocimiento de principios democráticos liberales $[\ldots] " 7$. Sin embargo, pudiera parecer que no es tanto el reconocimiento a un determinado régimen democrático sino el reconocimiento que nos ofrece ese régimen, el que nos torna ciudadanos y, por ende, sujetos de justicia. Ser reconocidos por un régimen jurídico como ciudadanos es lo que nos concede el estatus de sujetos capaces de reclamar y ser beneficiarios de justicia. No obstante, debemos examinar quién tiene derecho a plantear reivindicaciones y recibir reparaciones, es decir, quién

$\overline{7}$ Mouffe, C., "Ciudadanía democrática y comunidad política", El retorno de lo político, Barcelona, Paidós, 1999 , pp. 96. 
puede ser considerado sujeto de justicia. La admisión y exclusión de un sujeto al marco jurídico ha formado parte de la teoría de la justicia que se circunscribía a los límites nacionales, donde el espacio para la duda de quién podía formular reclamaciones de justicia era bastante limitado. De hecho, la participación en el marco estaba estrechamente ligada con la ciudadanía de una sociedad política definida por sus límites territoriales. En este sentido, estos mismos límites trazan la diferencia entre miembros y extranjeros, entre identidad (nacional) y diferencia (extranjera), es decir, entre quién es reconocido bajo el acuerdo de membresía y quién no. Como subraya Benhabib a lo largo de su obra, existe una tensión entre la declaración de derechos humanos y la soberanía de los estados nacionales a defender sus fronteras y controlar quiénes entran. Desde posiciones neokantianas redistributivas, podría sugerirse que las reparaciones de justicia no tendrían por qué hacerse desde el sujeto de justicia sino atendiendo al qué, es decir, a la necesidad de redistribuir las riquezas con los Estados más empobrecidos. Sin embargo, una teoría de la justicia que pretenda dar respuesta a los problemas de un mundo globalizado no puede simplemente atender a una justa distribución de los recursos manteniendo el esquema de ciudadanía nacional, sino que debe también prestar atención a lo que Benhabib llama una membresía justa. Existe, empero, un impedimento más que debemos considerar para el desarrollo de una teoría global de la justicia, y no es otro que el proceso globalizatorio. El problema de la membresía justa debe encuadrarse en pensar quién puede ser sujeto de justicia no solo dentro de los marcos nacionales sino a través de la sujeción a prácticas globales que lo afectan. Ahora sí, es el momento de meter en discusión el propio marco que delimita a los sujetos de justicia.

El modelo westfaliano presupone una única autoridad que ejerce su poder en un territorio delimitado. Sin embargo, "el Estado-nación es demasiado pequeño para gestionar los problemas económicos, ecológicos, inmunológicos e informativos creados por el nuevo medio, y al mismo tiempo es demasiado grande para dar lugar a las aspiraciones de movimientos sociales y regionalistas motivados por cuestiones de identidad" ${ }^{8}$. La dificultad para establecer un diálogo entre dentro y fuera, entre Estado-nacional y poderes supranacionales, encuentra en la teoría de la justicia otro tipo de formulación a través del quién de la justicia. ¿Qué sucede cuando el marco de los Estado no puede dar solución a los reclamos que sus ciudadanos demandan?, ¿qué sucede con aquellos sujetos que de hecho habitan esos límites nacionales y no son reconocidos como genuinos sujetos de justicia?

La disputa por el quién en la teoría de la justicia debe enmarcarse en una reflexión que incluya los flujos económicos y políticos globales para, de esta forma, poder re-

${ }^{8}$ Benhabib, S., "Crisis de territorialidad” Los derechos de los otros. Barcelona, Gedisa, 2005, p. 16. 
construir el sujeto de justicia en este contexto globalizado. Partiendo de esta necesidad de colocar al sujeto de justicia en el centro del debate en torno a la globalización, el trabajo de Fraser apunta a la "representación fallida político-ordinaria" para señalar "las injusticias políticas que surgen en el seno de una comunidad política, en la que las fronteras y la condición de miembro están ampliamente asumidas como estables"'. En este caso, el marco jurídico niega a algunos individuos su condición de sujetos para reclamar justicia, así como para acceder a los servicios que dicha comunidad ofrece al resto de habitantes. En este sentido, diremos que negar a algunos hombres participar como pares en la vida político-social de la comunidad, genera, desde el sistema político, injusticias de primer y segundo orden. De primer orden porque impiden a estos sujetos acceder a las demandas redistributivas o de reconocimiento, de segundo orden por el desplazamiento de sujetos de justicia que supone negar la participación en el marco político que se habita. Esta constitución como no miembros supone un perjuicio para los individuos apartados a los márgenes de la comunidad política, pues impide que sean tenidos en cuenta dentro de esta sociedad. Pensemos, además, en cómo influyen estructuras de gobernación que no se encuentran en los límites fronterizos de un país pero que de hecho afectan a la vida de sus ciudadanos. El descentramiento del sujeto de justicia sufre graves agravios al no poder participar en todas las instituciones que lo afectan. En este sentido, parece que la teoría de la justicia debe reformular el principio de la condición de miembro, hasta ahora empleado para delimitar quiénes podían tener acceso a las demandas de primer orden, principalmente de desde el plano social a través del reconocimiento y desde el económico desde las medidas redistributivas. Ya no basta con apelar a criterios de pertenencia política: ni en base al reconocimiento de una jurisdicción común ni mucho menos apelando a la ciudadanía o nacionalidad compartida. Desde que tenemos estructuras supranacionales que "no pertenecen al «espacio de los lugares", sino que ejercen su poder en el "espacio de los flujos»" 10 , no podemos seguir enmarcando la justicia desde los límites nacionales. Sin embargo, "las prácticas de inclusión y exclusión siempre están sujetas a cuestionamiento desde el punto de vista de la conversación moral infinitamente abierta" ${ }^{11}$. Por lo tanto, tenemos que encontrar también una respuesta al cómo del quién, es decir, en base a qué criterios vamos a demarcar el sujeto de justicia. Lo que podría derivar de esta problemática, es el planteamiento del principio del humanismo, según el cual el criterio delimitativo de la justicia vendría dado por criterios que remiten al ser humano. Bajo este principio, podríamos sencillamente finiquitar la cuestión remitiéndonos a los rasgos que compartimos los seres humanos, es decir, argumentar que

\footnotetext{
9 Fraser, N., Escalas de justicia, op. cit., p. 121.

${ }^{10}$ Fraser, N., Escalas de justicia, op. cit., p. 53.

11 Benhabib, S., Los derechos de los otros, op. cit., p. 22.
} 
todos los seres humanos, por el mero hecho de serlos, merecen tener un espacio en la conversación moral en torno a una cuestión de justicia. A pesar de que este principio pueda parecernos seductor, debemos señalar la problemática que entraña por su extremado nivel de abstracción y superficialidad que, aun sin quererlo, podría hacernos olvidar relaciones de poder en el seno de las interactuaciones entre humanos. Además, tenemos que tener presente la distinción entre moralidad y legalidad para no caer en una crítica simplemente moral, pues en última instancia lo que se pretende poner en cuestionamiento es la fundamentación legal de una normativa que afecta a varios sujetos o grupos. Precisamente para huir de "(...) la talla única de la humanidad global, [que] excluye la posibilidad de que diferentes cuestiones requieran diferentes marcos o escalas de justicia" ${ }^{22}$, Fraser plantea otro principio. Éste remite al nivel de sujeción al que se encuentran los individuos respecto a una estructura de poder. El «principio de todos los sujetos [a]» (all-subjected principle), indica que el marco de justicia no debería circunscribir a los sujetos a un marco nacional sino a una estructura de gobernación por la cual los sujetos se ven afectados, independientemente de la residencia que tengan los mismos. En otras palabras, la capacidad de plantear situaciones de injusticia en busca de su subsanación, no debería acotarse a los límites fronterizos de un país puesto que las instituciones supranacionales consiguen afectar a distintos grupos en diferentes partes del mundo. La negación de participación a algunos sujetos para reclamar justicia, nos deja como resultado el problema del enmarque que mencionábamos al principio y que es central para la teoría de la justicia contemporánea.

Por lo tanto, según el planteamiento anterior, para Fraser, una cuestión estaría justamente enmarcada cuando todos los sujetos afectados por una estructura de gobernación determinada estuvieran en igualdad de condiciones para formular reclamos y recibir la misma consideración. Sin embargo, los reclamos pueden venir desde diferentes países afectados por una misma institución supranacional como pueden ser la Organización Mundial del Comercio o el Fondo Monetario Internacional y, en estos casos, vale preguntarse: ¿cómo hacer para que todos los sujetos afectados en diferentes Estados del mundo reciban el mismo trato?

\section{De la transnacionalización de los mercados a la transnacionalización de la esfera pública}

EN PRIMER LUGAR, tenemos que examinemos cuál es el papel de la esfera pública y de los movimientos sociales. Por un lado, la esfera pública ha sido conceptualizada

${ }_{12}$ Fraser, N., Escalas de justicia, op. cit., p. 125. 
como "un espacio destinado a la formación comunicativa de opinión pública" 13 . Por otro lado, en torno a la teoría de la justicia han surgido principalmente dos oposiciones respecto a los movimientos sociales. Estos han sido entendidos por una gran parte del análisis de los años sesenta como la expresión colectiva en términos de respuestas agresivas ante la frustración que sentían los individuos por no ver cumplidas sus expectativas. Por ejemplo, Davies en su libro Violence in America subraya cómo este tipo de respuestas se dan ante el fin inesperado de períodos de bonanza económica. En este artículo se va a defender una posición que no tenemos tiempo de argumentar, pero que es aceptada desde los años ochenta por gran parte de la academia que estudia los movimientos sociales, que sostiene que estos funcionan como mecanismos colectivos para expresar la preocupación o el conflicto ante medidas adoptadas desde el ámbito político.

La dificultad reside en cuánta legitimidad pueden tener los movimientos sociales y la esfera pública como altavoces de aquellos sujetos marginados y, en última instancia, cómo se les otorga el estatus de movimientos reclamadores de justicia. Por un lado, Honneth y Calhoun han criticado el estatus del movimiento social por entender que estos espacios podían excluir aquellos reclamos provinientes de grupos que todavía no han sido reconocidos como reclamadores de política de identidad. Frente a esta posición, encontramos a Nancy Fraser o a Donatella della Porta, quienes subrayan la importante labor que tienen los movimientos sociales a la hora de reclamar reparaciones de injusticia, especialmente en aquellos casos donde las demandas no encuentran un espacio adecuado para narrarse. Cuando el reconocimiento institucional falla y desplaza a espacios periféricos a algunos sujetos que no tienen cómo exigir una revaluación de su estatus como ciudadanos, los movimientos sociales entran en juego. Podemos encontrar diversos movimientos a lo largo de la historia, pero por la extensión que puede tener este artículo, nos centraremos en aquellos que tienen que ver con la descentralización del sujeto en un mundo globalizado y su imposibilidad para reclamar justicia dentro de los marcos nacionales. Efectivamente, presenta un problema de enmarque la constitución de movimientos sociales cuyos reclamos ya no pueden continuar restringidos al nivel nacional, puesto que sus demandas pretenden tener como receptor instituciones supranacionales. Precisamente, los movimientos antiglobalización o, como decía Klein al entonces presidente de la Unión Europea Guy Verhofstadt, movimientos internacionalistas, han tratado de poner de relieve el insondable impacto de la internacionalización del neoliberalismo y los organismos supranacionales en la vida

${ }^{13}$ Fraser, N., "Transnationalizing the Public Sphere: On the legitimacy and efficacy of public opinion in a post-westphalian world", Transnationalizing the public sphere, Cambridge, Polity Press, 2014, p. 8. 
de las personas, manteniendo como objetivo de sus reclamos a esas instituciones transnacionales. "Al debatir este modelo, no estamos poniendo en tela de juicio el comercio de mercancías y servicios a través de las fronteras, sino los efectos mundiales de la profunda empresarialización, la forma en que «lo público» está siendo transformado y reorganizado -recortado, privatizado, desregulado- bajo la admonición de la competitividad en el sistema comercial mundial" ${ }^{14}$. En este contexto, Klein enfatiza el papel de los movimientos sociales en estas luchas de segundo orden que versan sobre el des-enmarque.

Entendemos los movimientos sociales como comportamientos colectivos que producen cambios culturales, ampliando horizontes de justicia que hasta entonces quedaban invisibilizados o desplazados. La capacidad de influencia de los movimientos sociales, acciones colectivas y protestas en los procesos políticos no debe ser subestimada. "Los movimientos sociales despliegan nuevas dimensiones de la justicia cuando consiguen establecer como plausibles reivindicaciones que transgreden la gramática establecida de la justicia normal, que, vista restropectivamente, mostrará haber ocultado la marginación padecida por sus miembros" ${ }^{15}$.

Sin embargo, debemos preguntarnos cuál es el papel de la esfera pública, hasta ahora delimitados al espacio nacional, en la era de la globalización, a la hora de poner de relieve nuevas problemáticas. Fraser propone, siguiendo una línea habermesiana, la transnacionalización de la esfera pública. Dado que los conflictos se encuentran cada vez más deslocalizados como resultado de la incipiente capacidad de influencia de las entidades supranacionales, parece que las reclamaciones de justicia deberían hacerse en el mismo espacio de los flujos. En este sentido, los movimientos sociales han visto cómo necesitan de apoyo de otros movimientos sociales para poder intervenir en la toma de decisiones políticas. "A través de estas luchas se abre una problemática nueva: una problemática constituyente" ${ }^{16}$. Inventar (o reiventar) el espacio común apunta a un ejercicio de reapropiación de las reclamaciones de la justicia, que ya no deben ser resueltos en los límites nacionales, así como las decisiones que generan las situaciones de conflictos no son tomadas dentro de esas fronteras. Los movimientos sociales generan nuevos espacios de diálogo y muestran lugares de resistencia común que son difícilmente localizables. Frente a la de-subjectivación o individuación del sujeto, los movimientos sociales proponen una reconstrucción de la población como sujeto colectivo de lucha.

\footnotetext{
${ }_{14}$ Klein, N., "Poner las reglas y saltáserlas", Vallas y Ventanas, Barcelona, Paidós, 2002, p. 95.

15 Fraser, N., Escalas de justicia, op. cit., p. 115.

${ }^{16}$ Guattari, F.; Negri. A., "Reapropiaciones del espacio público", Las verdades nómadas \& Intellect, poder constituyente, comunismo. Madrid, Akal, 1999, pp. 198.
} 
Habermas señala la legitimidad normativa y la eficacia política de la esfera pública como dos criterios que debemos tener en cuenta a la hora de evaluar el estatus de la esfera pública. Puesto que el término esfera pública en la propuesta habermesiana está extremadamente ligado al Estado westfaliano, al menos tal y como está desarrollado en Historia y crítica de la opinión pública: La transformación estructural de la vida pública, en lo que continúa mantendremos los criterios de validez aplicados no solo a la esfera pública sino también a las expresiones más concretas en forma de movimientos sociales. Los criterios empleados por Fraser de legitimidad y eficacia en la esfera pública transnacional nos sirven para pensar el papel de estos movimientos sociales en un mundo globalizado. Los movimientos sociales presentan un genuino interés para la teoría de la justicia “(...) porque hacen que se desarrollen los objetivos y los comportamientos de masas ligados a las nuevas contradicciones de la reestructuración y porque comienzan a constituir una sucesión de experiencias, de momentos de ruptura y/o negociaciones absolutamente originales" ${ }^{17}$. Sin embargo, necesitamos mantener los criterios propuestos en primer lugar por Habermas y mantenidos en la teoría de Fraser para depositar la confianza en su capacidad transformadora frente a los actuales retos que propone la globalización. Por un lado, la legitimidad remite al quién participa en esa opinión pública que va a negociar y participar en la reconstrucción del mundo en la era postwestfaliana. "En efecto, la teoría sostiene que la legitimidad de la opinión pública es función de dos características analíticamente distintas del proceso comunicativo, a saber, la extensión de su inclusividad y el grado en que hace real la paridad participativa" ${ }^{18}$. La legitimidad de esa formación y, en última instancia, de los reclamos que haga, viene dada en este esquema por la inclusión de sus miembros, es decir, quién puede participar en estos debates y, por otro lado, por el cómo se hace, es decir, si los interlocutores pueden participar en igualdad de condiciones en estos espacios. Para la aplicación de este criterio observamos cómo los movimientos sociales y la opinión pública necesitan sufrir el mismo proceso de trasnacionalización que de hecho ya han pasado aquellas estructuras con las que se pretende entablar un diálogo. Diremos, entonces, que la opinión pública formada con independencia del criterio demarcativo de ciudadanía que cumpla el requisito de inclusión y paridad participativa, es legítima. Por otro lado, el segundo criterio para la opinión pública o un movimiento social particular, es la eficacia. Fraser señala que ésta se expresa como eficaz cuando consigue tener algún tipo de incidencia en el poder público al que se enfrenta. Sin embargo, tener eficacia no conlleva necesariamente la aceptación de las reclamaciones expuestas

\footnotetext{
${ }_{17}$ Guattari, F.; Negri. A., "Luchas sociales y control sistémico", Las verdades nómadas \& Intellect, poder constituyente, comunismo. op. cit., pp. 189.

18 Fraser, N., Escalas de justicia, op. cit., p. 175.
} 
por parte de ese poder público, es decir, puede darse la negación de las mismas, pero, no obstante, haber sido escuchadas. La eficacia del movimiento que pretende reparar alguna situación de injusticia, por otro lado, se debe medir a largo plazo en función de la presión que consiga tener para establecer un diálogo de iguales.

Finalmente, es importante señalar que dada la diversidad de los problemas y de las personas sujetas a esas estructuras de gobernación, los acuerdos no pueden sino ser siempre parciales, temporales y sujetos a revisión. "Lejos de proporcionar el final, solución racional al problema de la justicia -que en la democracia moderna está condenada a permanecer como pregunta permanente y sin resolver-, la justicia como equidad es sólo una de las posibles interpretaciones de los principios de igualdad y de libertad" 19 .

Tenemos la ardua tarea de pensar cómo podría restructurarse y redefinirse el sujeto de justicia a través de un proceso que tenga como eje la paridad participativa que propone Fraser. Si asumimos el marco transnacional que venimos planteando a lo largo del artículo, parece que la respuesta por parte de los movimientos sociales también debería hacerse desde el campo supranacional, lo cual no deja libre de dificultades la tarea. Articular un movimiento social amplio e inclusivo, en constante revisión que nos permita examinar, bajo una mirada crítica, nuestras prácticas de exclusión y los acuerdos a los que queremos llegar.

${ }_{19}$ Mouffe, C., El retorno de lo político, op. cit., p. 81. 


\section{REFERENCIAS BibLIOGRÁficas}

Fraser, N. y Honneth, A., ¿¿Redistribución o reconocimiento? Madrid, Morata, 2006.

Habermas, J. y Rawls, J., Debate sobre el liberalismo politico. Barcelona, Ediciones Paidós Ibérica, 1998.

Crouch, C., Coping with Post-Democracy. London, Fabian Society, 2000.

Fraser, N., Escalas de justicia. Barcelona, Herder, 2008.

Della Porta, D., Globalizzacione e Movimenti Sociali. Roma, Manifestolibri, 2003.

Mouffe, C., El retorno de lo político. Barcelona, Paidós, 1999.

Benhabib, S., Los derechos de los otros, Barcelona, Gedisa, 2005.

Klein, N., Vallas y Ventanas, Barcelona, Paidós, 2002.

Fraser, N., Transnationalizing the public sphere, Cambridge, Polity Press, 2014.

Guattari, F.; Negri. A., Las verdades nómadas \& Intellect, poder constituyente, comunismo, Madrid, Akal, 1999.

DOI: https://doi.org/10.15366/bp2019.22.003

Bajo Palabra. II Época. No 22. Pgs: 81-94 